\title{
Risk factors for the introduction and within-herd transmission of Mycobacterium avium subspecies paratuberculosis (MAP) infection on $\mathbf{5 9}$ Irish dairy herds
}

Cashman, W. ${ }^{1}$, Buckley, J. ${ }^{2}$, Quigley, T. ${ }^{3}$, Fanning, S. ${ }^{4}$, More, S. ${ }^{4}$, Egan, J. ${ }^{5}$, Berry, D. ${ }^{6}$, Grant, I. ${ }^{7}$ and O' Farrell, K. ${ }^{8}$

1 Riverstown Cross, Glanmire, Co. Cork

2 Veterinary Department, Cork County Council, County Hall, Cork

3 safefood, Food Safety Promotion Board, 7 Eastgate Avenue, Little Island, Co. Cork

4 School of Agriculture, Food Science and Veterinary Medicine, University College Dublin, Belfield, Dublin 4

5 Central Veterinary Laboratory, Backweston, Co. Kildare

6 Moorepark Research Centre, Fermoy, Co. Cork

7 Institute of Agri-Food and Land Use, Queen's University, David Keir Building, Stranmillis Rd., Belfast, Northern Ireland

8 Greenhill, Fermoy Co. Cork

Since 1994, Irish cattle have been exposed to greater risks of acquiring Mycobacterium avium subspecies paratuberculosis (MAP) infection as a consequence of the importation of over 70,000 animals from continental Europe. In recent years, there has been an increase in the number of reported clinical cases of paratuberculosis in Ireland. This study examines the prevalence of factors that promote the introduction and within-herd transmission of Mycobacterium avium subspecies paratuberculosis (MAP) on selected Irish dairy farms in the Cork region, and the association between these factors and the results of MAP screening tests on milk sock filter residue (MFR). A total of 59 dairy farms, selected using non-random methods but apparently free of endemic paratuberculosis, were enrolled into the study. A questionnaire was used to collect data about risk factors for MAP introduction and transmission. The MFR was assessed on six occasions over 24 months for the presence of MAP, using culture and immunomagnetic separation prior to polymerase chain reaction (IMS-PCR). Furthermore, blood samples from all entire male and female animals over one year of age in 20 herds were tested by ELISA. Eighteen (31\%) farms had operated as closed herds since 1994, 28 (47\%) had purchased from multiple sources and $14(24 \%)$ had either direct or indirect (progeny) contact with imported animals. Milk and colostrum were mixed on 51\% of farms, while $88 \%$ of farms fed pooled milk. Thirty (51\%) herds tested negative to MFR culture and IMS-PCR, 12 (20\%) were MFR culture positive, 26 (44\%) were IMS-PCR positive and seven (12\%) were both culture and IMS-PCR positive. The probability of a positive MFR culture was significantly associated with reduced attendance at calving, and with increased use of individual calf pens and increased (but not significantly) if mulitiple suckling was practised. There was poor agreement between MFR culture and MFR IMS-PCR results, but moderate agreement between MFR culture and ELISA test results. This study highlights a lack of awareness among Irish dairy farmers about the effect of inadequate biosecurity on MAP introduction. Furthermore, within-herd transmission will be facilitated by traditional calf rearing and waste management practices. The findings of viable MAP in the presence of known transmission factors in non-clinically affected herds could be a prelude to long-term problems for the Irish cattle and agri-business generally.

Key Words: culture, dairy farms, IMS-PCR, Johne's disease, milk filter, paratuberculosis, risk factor

Irish Veterinary Journal Volume 61 Number 7 464-467, 2008

Corresponding author:

William Cashman, Riverstown Cross, Glanmire, Co. Cork

Email: bmcash@gmail.com

\section{Introduction}

Since 1994, Irish cattle have been exposed to greater risks of acquiring Mycobacterium avium subspecies paratuberculosis (MAP) infection as a consequence of the importation of over 70,000 animals from continental Europe (O'Doherty et al., 2002; Good, 2004). The number of notified clinical cases of MAP increased annually from three in 1997 to 153 in 2003 (Good, 2004). Brady (2004) also found that in five MAP infected herds, all had imported heifers from continental Europe.

Purchase of infected cattle, periparturient cow-calf man- agement and exposure to faeces of infected animals are the main risk factors associated with MAP transmission between and within farms (Collins, 2003; Rossiter et al., 2004). Brady (2004) found that there was a deficit of information among herdowners regarding the relationship between biosecurity practices and the risk of acquiring MAP. This study examines the prevalence of factors that promote the introduction and within-herd transmission of MAP on selected Irish dairy farms in the Cork region and the association between these factors and the results of MAP screening tests on milk sock filter residue (MFR). 


\section{Materials and methods Study herds}

In this study, the herd was the unit of interest. In total, 96 dairy herds in the Cork region were selected, using non-random methods but apparently free of endemic paratuberculosis, for inclusion in the study. All were sent a questionnaire of MAP risk factors (see below), and responses were obtained from 59 farms. A MFR test was conducted on all 59 farms over a 24-month period for the presence of known zoonotic pathogens. On 20 of these 59 farms, ELISA testing was also conducted. Some aspects of this study have been reported previously (McKee et al., 2003; Murphy et al., 2005).

\section{A questionnaire of risk factors for MAP introduction and transmission}

A questionnaire was developed, examining risk factors for MAP introduction and within-herd transmission (Collins, 2003; Rossiter et al., 2004), including farm biosecurity, perinatal cow-calf management, colostrum and milk usage and general hygiene. Data were collected about current (2003) practices as well as historical (1994 to 2002) data particularly in relation to the purchase or importation of cattle to the farm. Baseline data on current farm structure, production and animal health management were also collected in the questionnaire. This questionnaire was sent to all 96 study farms

\section{Milk sock filter residue testing}

The MFR was assessed on six occasions over 24 months from the 59 herds (Sept 2001 to Aug 2003). Each sample was examined for the presence of MAP, as described previously (Grant et al., 1998). Briefly, MAP DNA was detected using culture and immunomagnetic separation prior to polymerase chain reaction (IMS-PCR). Milk sock filters are used in modern milking systems to trap debris, large particles of organic matter and foreign objects so that when raw milk enters the bulk tank it is free from visible dirt (MacMillan, 2002). Herds were classified as MAPpositive if the test results were positive on at least one of the six sampling occasions, and negative otherwise.

\section{ELISA testing}

During 2004, serum samples were collected from 2,333 cattle in 20 herds. These samples, which were collected as part of routine national brucellosis testing, include all entire male and female animals over one year of age on each farm on the day of sampling. These samples were forwarded to the Central Veterinary Laboratory, Abbottstown, for ELISA testing (Institut Pourquier, Montpellier, France). A herd was considered positive if at least one ELISA result was positive.

\section{Statistical analysis}

Data were managed in Microsoft Excel (Microsoft Corporation, Redmond, WA, USA) and analysed using SAS (SAS Institute Inc., Cary, NC, USA). The questionnaire and test data were initially reviewed for errors. Descriptive analyses were conducted using routine methods.
The authors considered the association between MFR result (dependent variable: 1 , at least one positive result; 0 , otherwise) and a range of risk factors for MAP introduction and withinherd transmission. Preliminary exploration of the data revealed quasi-complete separation of the data across some of the dichotomous explanatory variables. This, coupled with the relatively small data size, suggested that logistic regression using the unconditional likelihood function and asymptotic methods may not be appropriate. Therefore, exact univariate logistic regression using PROC LOGISTIC (SAS, 2005) was performed across all dichotomous explanatory variables. In all cases, the dependent variable was modeled as the probability of a positive culture result. A logit link function was assumed in all cases. When analysing the effect of the normally distributed proportion of calving observed, it was treated as continuous within individual univariate analyses. Polychotomous explanatory variables were treated as classifiable variables in the analysis. Based on the relatively small dataset size, it was not appropriate to undertake multivariate analyses. There were missing data in some questionnaires. Therefore, herds with missing values for a variable of interest were not included in the analysis of that variable.

Kappa values were calculated to assess the level of agreement between the MFR and ELISA test results. All test sensitivities and specificities are applied at herd level, with MFR culture test result being used as the base test against which other tests are compared.

\section{Results}

\section{Questionnaire results}

a. General

Questionnaires were returned from 59 herds (response rate $61.5 \%$ ). In 2003, the average 305-day milk yield for 55 herds was 6,885 litres, with an average (SD) butterfat and protein percent of $3.77(0.22)$ and $3.4(0.20)$, respectively. The average (SD) disposal/culling rate was $17 \%$ (9). Fifty-two per cent of cows were less than third lactation, $32 \%$ fourth to sixth and $16 \%$ were over sixth lactation.

\section{b. Biosecurity}

In the period 1994 to $2002,47(80 \%)$ of the 59 farms had purchased animals. Only 20\% of farms had operated as closed herds since 1994. Twenty-eight (47\%) had purchased from multiple sources, while 14 (24\%) had either direct or indirect (progeny) contact with imported animals.

During 2003, 68\% of herds purchased cattle and $19 \%$ had contact with imported or progeny of imported cattle. Twenty-seven per cent had animal-to-animal contact at boundaries, and $32 \%$ shared common water sources. Rabbits and foxes were present on almost $90 \%$ of farms.

c. Periparturient cow-calf management

Routine pre-calving udder clipping was carried out on $84 \%$ of farms, and on average (SD) $64 \%$ of calvings (32) were supervised. Eight farmers (14\%) reported that they observed all calvings but only one removed the calves immediately and three allowed suckling for over 24 hours. On nine 
farms (14\%), calves were allowed to suckle their dams for more than 24 hours, while multiple suckling occurred on five farms (8\%). Ninety-two per cent of farms fed an average of 10.8 litres of dam's colostrum by hand, and $27 \%$ fed pooled colostrum in the first 72 hours of life. Milk and colostrum were mixed on $51 \%$ of farms, while $88 \%$ of farms fed pooled milk. Only $25 \%$ fed milk substitute. Seventy-six per cent of farms used individual calf pens and the average weaning age was eight weeks.

\section{d. Animal waste and hygiene management}

On $56 \%$ of farms, slurry was spread on calf pasture and calves grazed adult pasture on $53 \%$ of farms. Contractors were used to spread slurry on $34 \%$ of farms. Faecal contamination of water troughs by adults was possible on $51 \%$ of farms. Pre-milking udder washing was carried out on $35 \%$ of farms all year, and on $35 \%$ during winter only.

\section{MFR test results}

The MFR culture results are presented in Table $\mathbf{1}$.

\section{Association between MFR test result and risk factors for introduction and within-herd transmission}

The probability of a positive MFR culture on-farm was significantly $(\mathrm{P}<0.05)$ reduced as the percentage of calvings attended increased. A three percentage unit decrease in the probability of a positive culture result is expected if the percentage of calvings attended was increased from the sample mean of $64 \%$ to $74 \%$. The odds of a positive MFR culture result while over seven times greater (95\% CI: 0.71 to 97.0 ) if multiple sucking was practised, was not significant $(\mathrm{p}>0.10)$. On farms where individual calf pens were used, the odds of a positive MFR culture was significantly $(\mathrm{P}<0.05)$ lower $(\mathrm{OR}=0.21$; 95\% CI: 0.04 to 1.0$)$. No significant association was found between colostrum pooling, udder washing, udder drying and use of teat dip and MFR culture result with P-values of $0.89,0.29,0.22$ and 0.54 , respectively. Neither herd size nor culling rate, when treated as continuous variables, significantly affected the probability of a positive MFR culture. None of the risk factors relating to biosecurity or animal waste and hygiene management was significantly associated with the probability of a positive MFR culture. The MFR IMS-PCR results were not significantly associated with any risk factors.

Table 1: Milk Filter Residue culture result for the 59 surveyed herds
\begin{tabular}{|l|l|l|}
\hline Culture positive & Culture negative & Total \\
\hline $12(20 \%)$ & $47(80 \%)$ & $59(100 \%)$
\end{tabular}

\section{Agreement between test results}

Among the 59 herds, 12 and 24 were MFR culture and MFR IMS-PCR positive, respectively. There was a poor level of agreement between these test results ( $\mathrm{kappa}=$ $0.16,95 \% \mathrm{CI}:-0.07$ to $0.39 ; \mathrm{p}=0.16)$. Among the 20 herds where ELISA testing was conducted, there was moderate agreement between the MFR culture and ELISA test results (kappa $=0.53,95 \% \mathrm{CI}: 0.21$ to $0.84 ; \mathrm{p}=0.007$ ). Of the 20 herds, eight were MFR culture positive. Each of these eight herds had ELISA-positive animal(s) (3 herds, 1 ELISA positive animal; 3 herds, 2; 2 herds, 3). Five of 12 MFR culture negative herds were also ELISA positive. No comparison between ELISA and combined MFR culture and IMS-PCR was possible due to small sample size. In total, 24 (1.0\%) of the 2,333 animals were ELISA positive.

\section{Discussion}

The 59 farms in this study were family-run enterprises typical of the region (Murphy et al., 2005). None had a history of endemic clinical MAP infection. The MFR culture of MAP in 12 of these herds, coupled with the extent to which transmission factors exist on the surveyed farms, is a cause for concern. The lack of awareness quoted by Brady (2004) regarding the effect of inadequate biosecurity is compounded by traditional calf rearing and waste management practices. Under these circumstances, it can be expected that MAP will spread subclinically for a variable period until the occurrence of clinical cases (Kennedy and Benedictus, 2001). The low usage of milk replacer, coupled with an eight-week weaning period during which pooled milk is fed, provide ideal opportunities for MAP transmission (Muskens et. al., 2003). Despite the small sample size in this study, the association between MAP transmission factors such as lack of calving supervision and prolonged suckling and MFR culture-positive herds is in agreement with similar studies in other countries (Kalis et al., 2004, Beaudeau et al., 2005). However, other factors such as purchase of cattle on the open market, udder washing and clipping and animal waste management were not found to be significantly associated with MFR culture result in this study. Experience from other countries (Groenendaal and Galligan, 2003) suggests that current animal management practices should be modified if transmission of MAP among and within Irish dairy herds is to be controlled.

Improving the Johne's disease status of individual infected herds depends on the identification and elimination of infected animals and breaking the cycle of transmission (Johnson-Ifearulundu and Kaneene, 1998). The most rapid and effective progress is achieved when repeated individual animal testing is used within a biosecure management programme (Johnson-Ifearulundu and Kaneene, 1999; Kalis et al., 2004; Rossiter et al., 2004). On-farm management such as calf removal at calving, milk feeding and management of animal waste (Beaudeau et al., 2005; Flynn et al., 2005) are critical components of any protocol. However, economic considerations will affect the choice of programme to be implemented (Groenendaal and Galligan 2003; Dorshorst et al., 2006).). Such programmes may well be easier to implement and provide a better return if integrated into a whole herd health management system which encompasses several economically important diseases.

Culture is a recognised method of identifying viable MAP organisms, even though its sensitivity is relatively low. The milk sock filter is preferable to bulk milk when testing for MAP, noting that the milk sock is used to filter milk from all animals in a dairy herd, including any faecal material. 
this approach, the chances of detecting the presence of MAP, and other pathogens, is maximised. Such organisms may arise in MFR from direct udder secretion or faecal contamination originating from the hide and teats during milking. Twenty per cent (12) of the surveyed herds in the study had culture positive MFR. IMS-PCR detects non-viable as well as viable MAP. This, coupled with a faster sample turn around time made it an attractive comparative test to use on MFR in this study. Positive IMS-PCR samples were detected in $44 \%$ (26) compared to $20 \%$ MFR culture positive herds.

In this study, among the $12 \mathrm{MFR}$ culture positive herds, each was positive at only one of the six testing events over the twoyear period. As found in other studies, this low repeatability could be due to losses in pre-culture processing, dilution of MAP, low disease prevalence, or few high shedding animals contributing to the samples. In addition, MFR samples are subject to the normal variation in the milking cow population, and possible variation in milking hygiene practices. Additionally, the present study found that none of the transmission risk factors were associated significantly with MFR IMS-PCR results. Such positive associations would be expected if MFR IMS-PCR was as specific as MFR culture. Based on these findings and those of other studies (Cousins et al., 1999), the use of PCR as the sole means of categorising herds for the presence of MAP could be open to question. However, any positive IMS-PCR result in a herd would warrant further investigation.

While this study was confined to a small and select group of herds within an intensive dairying area, the findings of viable MAP in the presence of known transmission factors in non-clinically affected herds could be a prelude to long-term problems for the Irish cattle and agri-business generally. Experience from other countries would suggest that the Irish agricultural industry would benefit from preventing MAP from becoming an endemic clinical disease. Should any future perceived or real connection between MAP and Crohn's disease lower public confidence in food safety, the benefit of early intervention would prove even more cost-effective.

\section{Acknowledgements}

The authors wish to acknowledge the advice and assistance of Mr Eugene Power and Mr Seamus Power of the Department of Agriculture, Fisheries and Food Regional Laboratory Service and the support of Professor M. Monaghan from the UCD School of Veterinary Medicine.

\section{References}

Beaudeau, F., Ledoux, D., Poumerol, N., Joly, A. and Seegers, H. (2005). Risk factors for Mycobacterium avium subspecies paratuberculosis infection in dairy herds in Brittany (Western France). In: E.J.B. Manning and S.S. Neilsen (eds), Proceedings of the 8th International Colloquium on Paratuberculosis, International Association for Paratuberculosis, Madison, Wisconsin. p. 255.

Brady, C. (2004). An epidemiological and pathological investigation of Johne's disease in Irish cattle. MVM thesis: University College Dublin Collins, M. T. (2003). Update on paratuberculosis. Irish Veterinary Journal 56 (12): 619-623.

Cousins, D.V., Whittington, R., Marsh, I., Masters, A., Evans, R.J. and
Kluver, P. (1999). Mycobacteria distinct from Mycobacterium avium subsp. paratuberculosis isolated from the faeces of ruminants possess IS900-like sequences detectable by IS900 polymerase chain reaction: implications for diagnosis. Molecular and Cell Probes 13: 431-442.

Dorshorst, N.C., Collins, M.T. and Lombard, J.E. (2006). Decision analysis model for paratuberculosis control in commercial dairy herds. Preventive Veterinary Medicine 75: 92-122.

Flynn, O., Egan, J., O Grady, D. and Good, M. (2005). Inactivation of Mycobacterium avium subsp. paratuberculosis (MAP) in bovine slurry. In: E.J.B. Manning and S.S. Neilsen (eds), Proceedings of the 8th International Colloquium on Paratuberculosis, International Association for Paratuberculosis, Madison, Wisconsin. p. 259.

Good, M. (2004). Proceedings of Conference on the role of veterinary practitioners in the control of Bovine Johne's Disease. Irish Department of Agriculture and Food.

Grant, I.R., Ball, H.J. and Rowe, M.T. (1998). Isolation of Mycobacterium paratuberculosis from milk by immunomagnetic separation. Applied and Environmental Microbiology 64: 3153-3158.

Groenendaal, H. and Galligan, D.T. (2003). Economic consequences of control programmes for paratuberculosis in midsize dairy farms in the United States. Journal of the American Veterinary Medical Association 223: 1757-1763.

Johnson-Ifearulundu, Y. and Kaneene, J.B. (1998). Management-related risk factors for $M$. paratuberculosis infection in Michigan, USA, dairy herds. Preventive Veterinary Medicine 37: 41-54.

Johnson-Ifearulundu, Y. and Kaneene, J. B. (1999). Distribution and environmental risk factors for paratuberculosis in dairy cattle herds in Michigan. American Journal of Veterinary Research 60: 589-596.

Kalis, C.H.J., Collins, M.T., Barkema, H.W. and Hesselink, J. W. (2004)

Certification of herds as free of Mycobacterium paratuberculosis infection: actual pooled faecal results versus certification model predictions. Preventive Veterinary Medicine 65: 189-204.

Kennedy, D. and Benedictus, G. (2001). Control of Mycobacterium avium subsp. paratuberculosis infection in agricultural species. Revue Scientifique et Technique-Office International Epizooties 20: 151-179.

MacMillan, S. (2002). Fail-safe filters. Dairy Farmer April issue, p. 36. McKee, R., Buckley, H.G., Buckley, J.F. and Fanning, S. (2003). Examination of in-line milk filters to detect Mycobacterium avium subsp. paratuberculosis at farm level. Proceedings of the 7th International Colloquium on Paratuberculosis, ed. R.A. Juste, M.V. Geijo and J.M. Garrido, p. 258-260. International Association for Paratuberculosis, Madison, Wisconsin.

Murphy, B. P., Murphy, M., Buckley, J.F., Gilroy, D., Rowe, M.T., McCleery D. and Fanning S. (2005). In-line milk filter analysis: Escherichia coli $\mathrm{O} 157$ surveillance of milk production holdings. International Journal of Hygiene and Environmental Health 208: 407-413.

Muskens, J., Elbers, A. R. W., van Weering, H.J. and Noordhuizen, J.P.T.M. (2003). Herd management practices associated with Johne's disease infection in Dutch dairy herds. Journal of Veterinary Medicine B 50: 372-377.

O'Doherty, A., O Grady D., O Farrell, K, Smith, T. and Egan, J. (2002). Survey of Johne's disease in imported animals in the Republic of Ireland. Veterinary Record 150: 634-636.

Rossiter, C. A., Hansen, D., Hutchinson, L.J. and Whitlock, R.H. (eds) (2004). Johne's Disease - Prevention and Control in Dairy Herds. A Manual for Veterinarians. www.johnes.org/handouts/files/Rossiter_ workbook.pdf. Last accessed July 2007. 